\title{
EBOLA VIRUS DISEASE AND THE SEARCH FOR A CURE
}

\author{
Andre C. Kalil ${ }^{1}$
}

\begin{abstract}
Ebola virus disease (EVD) was first identified in 1976 in Yambuku, Zaire (now the Democratic Republic of Congo), and is caused by an RNA virus in the filovirus family (Feldmann \& Geisbert). The current strain circulation in West Africa is very similar to the original strain ( $>95 \%$ homology). The origin of the current outbreak remains unknown, but it is suspected to be from an animal reservoir with intermediary species (Fauci). Randomized clinical trials with adaptive design are ongoing to evaluate potential new therapies for EVD.
\end{abstract}

Keywords: Infectious diseases; Ebola virus disease; EVD

Ebola virus disease (EVD) was first identified in 1976 in Yambuku, Zaire (now the Democratic Republic of Congo), and is caused by an RNA virus in the filovirus family ${ }^{1}$. The current strain circulating in West Africa is very similar to the original strain ( $>95 \%$ homology). The origin of the current outbreak remains unknown, but it is suspected to be from an animal reservoir and may involve intermediary species ${ }^{2}$.

EVD has affected more than 27,000 people in West Africa, mostly in Liberia, Sierra Leone, and Guinea; the case-fatality rate has ranged from $20-80 \%$, with an overall average mortality around $50 \%{ }^{3}$. Transmission occurs through direct contact with infected bodily fluids and may be associated with mucosal surfaces, skin breaks, and parenteral routes. Contact with patients with suspected or confirmed EVD seems to be the strongest independent predictor, based on a recent study ${ }^{4}$. The incubation period varies from 2-21 days, but most cases present within the first 7 days. Fever and fatigue are the most common initial symptoms in greater than $90 \%$ of patients; in addition, arthralgia, myalgia, headache, sore throat, anorexia, and rash can be present. After a few days, the gastrointestinal symptoms start with nausea, vomiting, abdominal pain, and severe diarrhea, with up to 5-10 liters of fluid loss per day. At this stage, hypovolemic shock and renal failure may develop quickly, followed by multi-organ failure. Coagulation disturbances and bleeding have been less common in the current outbreak and tend to occur in the late phases of the disease. Laboratory abnormalities include hyponatremia, hypokalemia, hypophosphatemia, hypomagnesemia, lymphocytopenia, thrombocytopenia, raised liver enzymes, metabolic acidosis, creatinine elevation, increased international normalized ratio and d-dimers ${ }^{1,2}$.

The current outbreak started in April of 2014, and despite substantial decrease in the number of new cases in the last few months, the outbreak is still ongoing in Sierra Leone and Guinea ${ }^{3}$. The reasons for such a large outbreak in this specific region are multifactorial: 1) decades of geopolitical conflicts; 2 ) close country borders with easy mobility among these countries; 3 ) poor healthcare infrastructure (e.g. limited availability of protective equipment, as well as of isolation beds); 4 ) restricted number of healthcare professionals (i.e. physicians, nurses, assistants, microbiology and laboratory professionals);
Clin Biomed Res. 2015;35(2):83-85

1 Infectious Diseases Division, University of Nebraska Medical Center. Omaha, NE, USA.

Corresponding author: Andre Kalil

E-mail: akalil@unmc.edu Transplant ID Program, Infectious Diseases Division, Department of Internal Medicine, University of Nebraska Medical Center Director

985400, Nebraska Medical Center. 68198-5400, Omaha, Nebraska, USA. 
5) traditional practices that include bathing of corpses before burial; 6) concurrent healthcare outbreaks and challenges with other infectious diseases such as malaria, HIV, and typhoid fever.

Twenty-six patients with confirmed EVD have been evacuated to the European Union and USA since the beginning of the outbreak. In the USA there are three medical centers that can receive patients from Africa who are diagnosed with EVD: University of Nebraska Medical Center (Omaha, Nebraska), Emory University (Atlanta, Georgia), and the National Institutes of Health (Bethesda, Maryland). What these centers have in common is a biocontainment unit comprised of highly trained medical personnel and an infrastructure equipped with specifically designed space and materials to deal with highly contagious infectious diseases.

Isolation of patients with exposure to Ebola and new onset fever maintains public safety and provision of proper personal protective equipment (PPE) gives the caregiver the confidence to treat this disease. The most important factor to maintaining isolation is careful attention to the donning and doffing of PPE. All caregivers going into the unit had to change into scrubs and put on the PPE one would use for universal precautions including a surgical mask, isolation gown and gloves. Everyone wore slip-on rubber shoes with shoe covers that were removed upon leaving the unit and dipped in a bleach solution. Entering the 'hot zone' (patient location) required additional PPE and another team member to observe the process to make sure there were no breaks in the PPE. Doffing was also a two-person job requiring decontamination at each step with alcohol hand gel and new gloves ${ }^{5}$. Resources from the University of Nebraska Biocontainment Unit are available outlining each step of this process ${ }^{6}$.

Our biocontainment unit at the University of Nebraska Medical Center took care of three patients diagnosed with Ebola. Optimized supportive care was the most immediate and standard treatment offered to all patients. It included the placement of peripheral and central lines, volume replacement with balanced solutions, electrolytes corrections, laboratory and microbiological monitoring, verification of RNA viremia by polymerase chain reaction techniques, airborne and contact isolation techniques. The acuity of the patients dictated that a team approach to the physician care of these patients be taken. The intensivists obtained central venous access, managed fluid and electrolytes and nutrition as well as any critical care issues that arose. The infectious disease specialists worked on the treatment of the virus, health care worker protection, equipment decontamination, and investigational new drug applications for the Food and Drug Administration ${ }^{5}$.

In addition to the optimized supportive care, the patients admitted to the University of Nebraska Medical Center received several investigational drugs as compassionate use through the FDA: convalescent plasma, small interfering RNA nanoparticles, polymerase inhibitors, and monoclonal antibodies ${ }^{7,8}$. The problem with uncontrolled administration of investigational drugs is that inferences about the effects of these potential therapies are very difficult to make since many factors can contribute to the survival or demise of these patients with EVD: patients' own immune system, quality of supportive care, and effects of specific investigational drugs ${ }^{7}$. The only way to make more reliable inferences about efficacy is by providing these investigational drugs in the setting of randomized controlled studies. Fortunately, the National Institutes of Health (Bethesda, Maryland), in conjunction with the University of Nebraska Medical Center, Emory University, and several hospitals in both Liberia and Sierra Leone, designed and started a randomized clinical trial to test several investigational drugs sequentially based on an innovative trial design denominated Bayesian adaptive design?. This randomized clinical trial is currently ongoing and enrolling patients in order to evaluate the first drug: the monoclonal antibody combination called ZMapp ${ }^{10}$. The adaptive design requires a smaller number of patients than conventional design to reach efficacy and safety endpoints. Once the conclusion of benefits (or lack of) is reached, the second investigational drug will be automatically started without any clinical trial interruption. If ZMapp proves to be beneficial, then this drug will become incorporated as part of the standard of care to be utilized in the next study step (sequential administration of the second investigational drug to be tested); if ZMapp does not cause benefit or causes harm, this drug will be removed from the trial, and then the second investigational drug will be tested against the same standard of care used in the first part of the study. Of note, both the American and African sites that are participating in this new clinical trial will not offer these investigational drugs to their patients outside the context of the clinical trial. We believe that this robust and appropriate trial design approach will maximize the chances to discover new cures for this devastating disease, as well as to be prepared for future outbreaks.

\section{Conflicts of interest}

None to declare. 


\section{REFERENCES}

1. Feldmann H, Geisbert TW. Ebola haemorrhagic fever. Lancet. 2011;377(9768):849-62. http://dx.doi. org/10.1016/S0140-6736(10)60667-8. PMid:21084112.

2. Fauci AS. Ebola--underscoring the global disparities in health care resources. N Engl J Med. 2014;371(12):1084-6. http://dx.doi. org/10.1056/NEJMp1409494. PMid:25119491.

3. World Health Organization (WHO). Ebola Roadmap Situation Report. [cited 2015 June 21]. Available from: http://apps.who.int/ebola/ebolasituation-reports.

4. Levine AC, Shetty PP, Burbach R, Cheemalapati S, Glavis-Bloom J, Wiskel T, et al. Derivation and internal validation of the Ebola prediction score for risk stratification of patients with suspected Ebola Virus Disease.
Ann Emerg Med. 2015. pii: S01960644(15)00217-6.

5. Johnson DW, Sullivan JN, Piquette CA, Hewlett AL, Bailey KL, Smith $\mathrm{PW}$, et al. Lessons learned: critical care management of patients with Ebola in the United States. Crit Care Med. 2015;43(6):115764. http://dx.doi.org/10.1097/ CCM.0000000000000935. PMid:25756410.

6. The Nebraska Ebola Method - for Clinicians. [cited 2015 Jun 20]. Available from: https://www.unmc.edu/ cce/catalog/online/ebola/index.html.

7. Florescu DF, Kalil AC, Hewlett AL, Schuh AJ, Stroher U, Uyeki TM, et al. Administration of brincidofovir and convalescent plasma in a patient with Ebola Virus Disease. Clin Infect Dis. 2015;civ395. PMid:25991468.
8. Kraft CS, Hewlett AL, Koepsell S, Winkler AM, Kratochvil CJ, Larson L, et al. The Use of TKM-100802 and Convalescent Plasma in 2 Patients With Ebola Virus Disease in the United States. Clin Infect Dis. 2015:civ334. PMid:25904375.

9. Kalil AC, Sun J. Bayesian methodology for the design and interpretation of clinical trials in critical care medicine: a primer for clinicians. Crit Care Med. 2014;42(10):226777. http://dx.doi.org/10.1097/ CCM.0000000000000576. PMid:25226118.

10. U.S. National Institutes of Health (NIH). Putative Investigational Therapeutics in the Treatment of Patients With Known Ebola Infection. [cited 2015 Jun 21]. Available from: https://clinicaltrials.gov/ct2/show/ NCT02363322?term=zmapp\&rank=1. 